\title{
N92-21906
}

\section{OSSE OBSERVATIONS OF THE CRAB PULSAR}

\author{
M. P. Ulmer; S. M. Matz; R. A. Cameron $\dagger$ \\ D. A. Grabelsky*, J. E. Grove; W. N. Johnson† G. V. Jung \\ R. L. Kinzer ${ }^{\dagger}$ J. D. Kurfess ${ }^{\dagger}$ M. D. Leising $\}^{\mathbb{I}}$ W. R. Purcell; M. S. Strickman ${ }^{\dagger}$
}

\begin{abstract}
We present preliminary results of the Compton Gamma Ray Observatory OSSE observations of the Crab pulsar. The pulsar energy spectra and light curves are in general agreement with previous observations, validating the OSSE pulsar data acquisition modes and data analysis algorithms. The data suggest that the spectrum of the pulsar varies throughout the light curve. The "interpulse" region has a slightly flatter spectrum in the $\sim 60-250 \mathrm{keV}$ region and a slightly steeper spectrum at higher energies than the two main pulses. We find no evidence for any lines in the spectra with a typical sensitivity of about $10^{-4}$ photons $\mathrm{cm}^{-2} \mathrm{~s}^{-1}$.
\end{abstract}

\section{INTRODUCTION}

The Oriented Scintillation Spectrometer Experiment (OSSE; Johnson et al. 1989) observed the Crab pulsar during instrument activation and also during observation period 1. In this paper we report on the preliminary analysis of the data acquired during viewing period 1 (17 May 1991 to 30 May 1991).

The OSSE pulsar data modes permit acquisition of time-tagged gamma-ray energy losses for the study of fast pulsars. The entire event stream for the detectors cannot be accommodated on an event-by-event basis in telemetry; consequently, the pulsar processing includes event selection and compression for telemetry formatting. The pulsar processing permits the definition of up to eight energy bands to be included in the transmitted pulsar data. These energy bands, as well as the rest of the pulsar data collection configuration, can be defined by OSSE mission operations activities and uploaded into the experiment via command. The pulsar data can therefore be optimized to the specific observing strategy and energy range of particular interest while limiting the event rate to that which can be handled in the OSSE telemetry.

\footnotetext{
- Northwestern University, Evanston, IL

INaval Research Laboratory, Washington DC

t NRC/NRL Resident Research Associate

Universities Space Research Association, Washington DC

"Clemson University, Clemson, SC
} 
Table 1: Crab pulsar ephemeris

\begin{tabular}{ll}
\hline \hline$\nu$ & $29.9493820573442 \mathrm{~s}^{-1}$ \\
$\dot{\nu}$ & $-3.77660 \times 10^{-10} \mathrm{~s}^{-2}$ \\
$\ddot{\nu}$ & $7.45 \times 10^{-21} \mathrm{~s}^{-3}$ \\
T0 & $8367.0(\mathrm{TJD})$ \\
RA & $5^{\mathrm{h}} 34^{\mathrm{m}} 31: 973$ \\
Dec & $22^{\circ} 00^{\prime} 52^{\prime \prime} 06$ \\
\hline
\end{tabular}

Gamma-ray events qualified as being in one of these eight energy bands are then processed in one of two modes: (1) event-by-event mode, where selected events are time-tagged, and both energy loss and arrival time of the event are transmitted in the telemetry, or (2) rate mode, where high time resolution rate samples are taken in each of the eight energy bands.

The event-by-event mode of pulsar data provides the highest time resolution for the study of fast pulsars. This mode time-tags events accurate to 0.125 milliseconds at its highest resolution, and transmits the arrival times, detector identifications, and encoded energy losses. Depending on the OSSE telemetry format, a maximum of $\sim 290$ events per second is supported in the event-by-event pulsar mode.

The pulsar rate mode can accommodate a much higher event rate but at the sacrifice of spectral resolution. This mode records the number of events in each of the defined energy bands at a specified sample frequency. The highest sample rate in this mode provides a resolution of 4 milliseconds. Sample times from $4 \mathrm{msec}$ to $512 \mathrm{msec}$ can be selected. In this mode OSSE can achieve its best sensitivity to a continuum flux.

The data presented here were collected in the 1 millisecond event-by-event (or EBE) mode, and various energy ranges were selected at different times for different detectors in order to cover nearly the entire $50 \mathrm{keV}-10 \mathrm{MeV}$ range with optimal energy resolution.

\section{ANALYSIS METHOD}

Using a version of TEMPO (Taylor and Weisberg 1989) and a position of the Crab pulsar based on an ephemeris provided by Taylor and Nice (private communication), we corrected the arrival time of the time-tagged photons to the solar system barycenter. Next, we epoch-folded the data using the relative phases of these events calculated from the ephemeris provided by Taylor and Nice (Table 1). The resulting light curve for the $\sim 60-$ $246 \mathrm{keV}$ energy range integrated over the observations made from TJD 8393.6 to 8399.5 is shown in Figure 1. In order to compare the phase of our pulse with that seen at radio frequencies, further phase corrections must be applied to the light curve (Taylor, private 


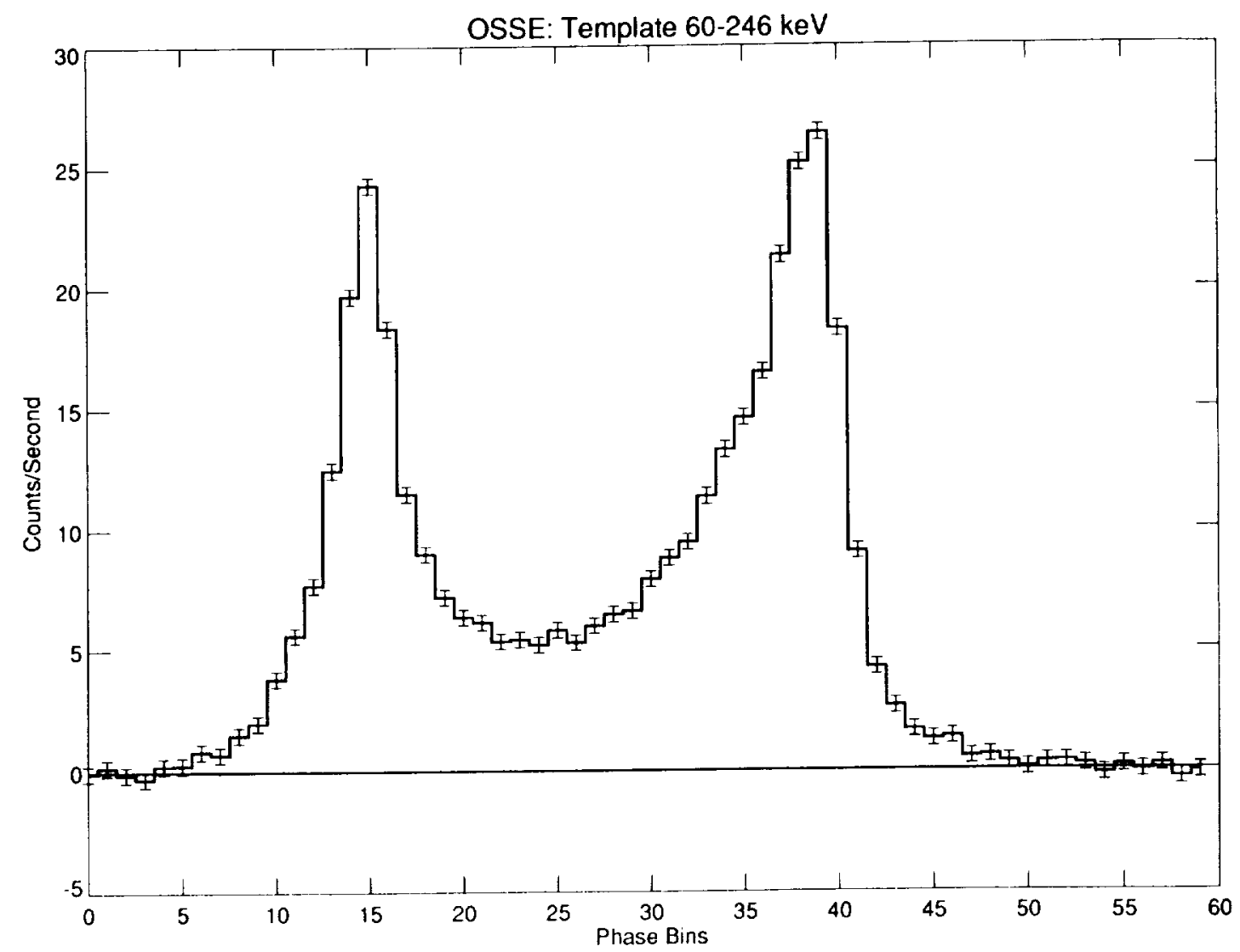

Figure 1: The average Crab pulsar light curve.

communication). However, even after applying these corrections, there is still a net offset of approximately 6 milliseconds between the two light curves. This offset is almost certainly not real, but is probably caused by insufficient accuracy in extrapolating the radio phase to infinite frequency. Further study of this problem is required.

\section{PRELIMINARY RESULTS}

In Figure 2 we show the light curves in several broad energy channels and in Figure 3 we show the hardness ratio as a function of phase. From these two figures we see that in the lower energy range, the spectrum of the interpulse region (phase bins 20 to 29) is flatter than the spectra of the pulse peaks (phase bins 14 to 19 and 35 to 41). This is in basic agreement with Knight (1982), Mahoney et al. (1984), and Agrinier et al. (1990). We have produced several preliminary spectra, but it is too early to report a detailed spectral comparison between the different regions. 


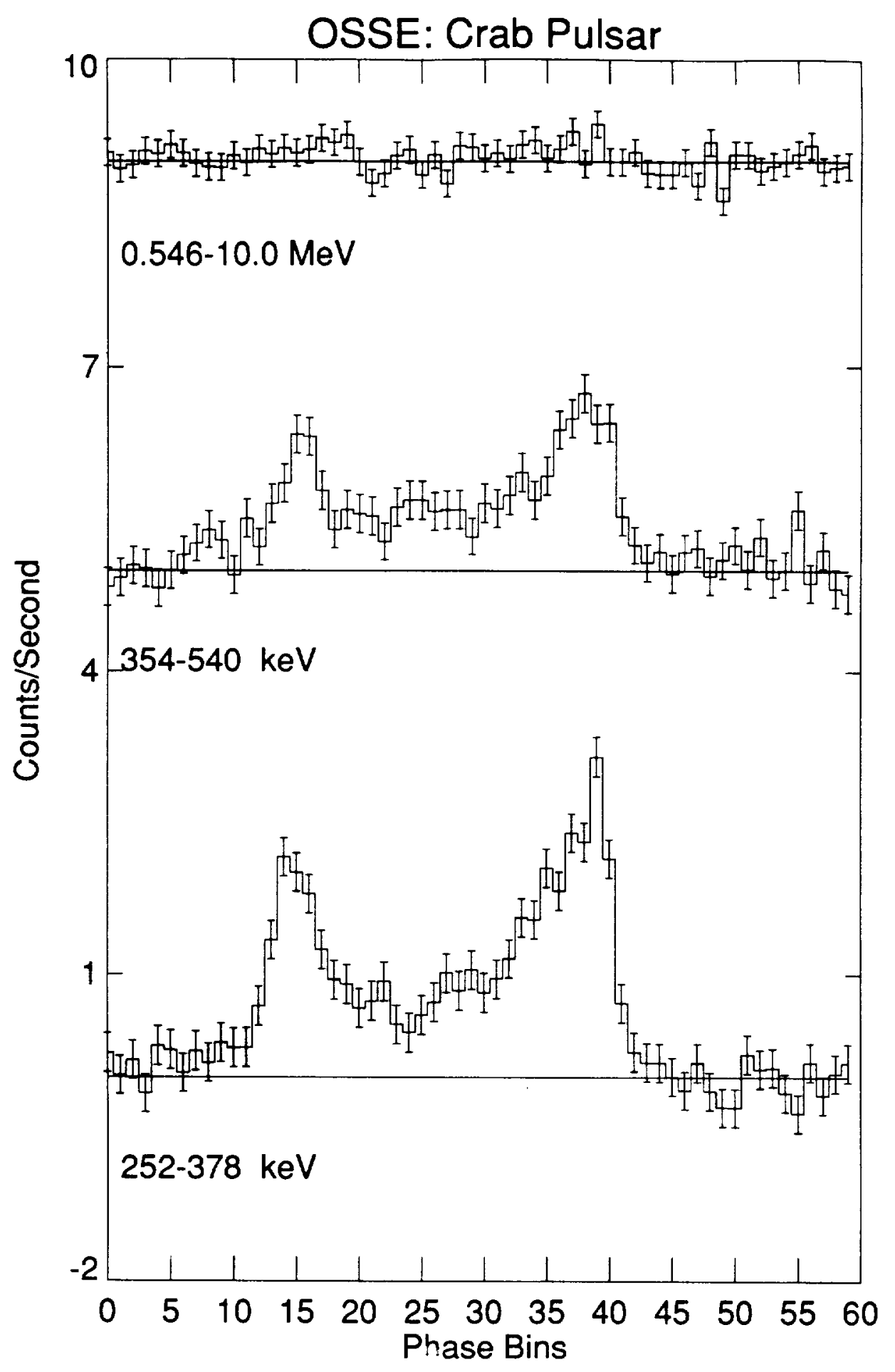

Figure 2: The Crab pulsar light curve in different energy ranges. 


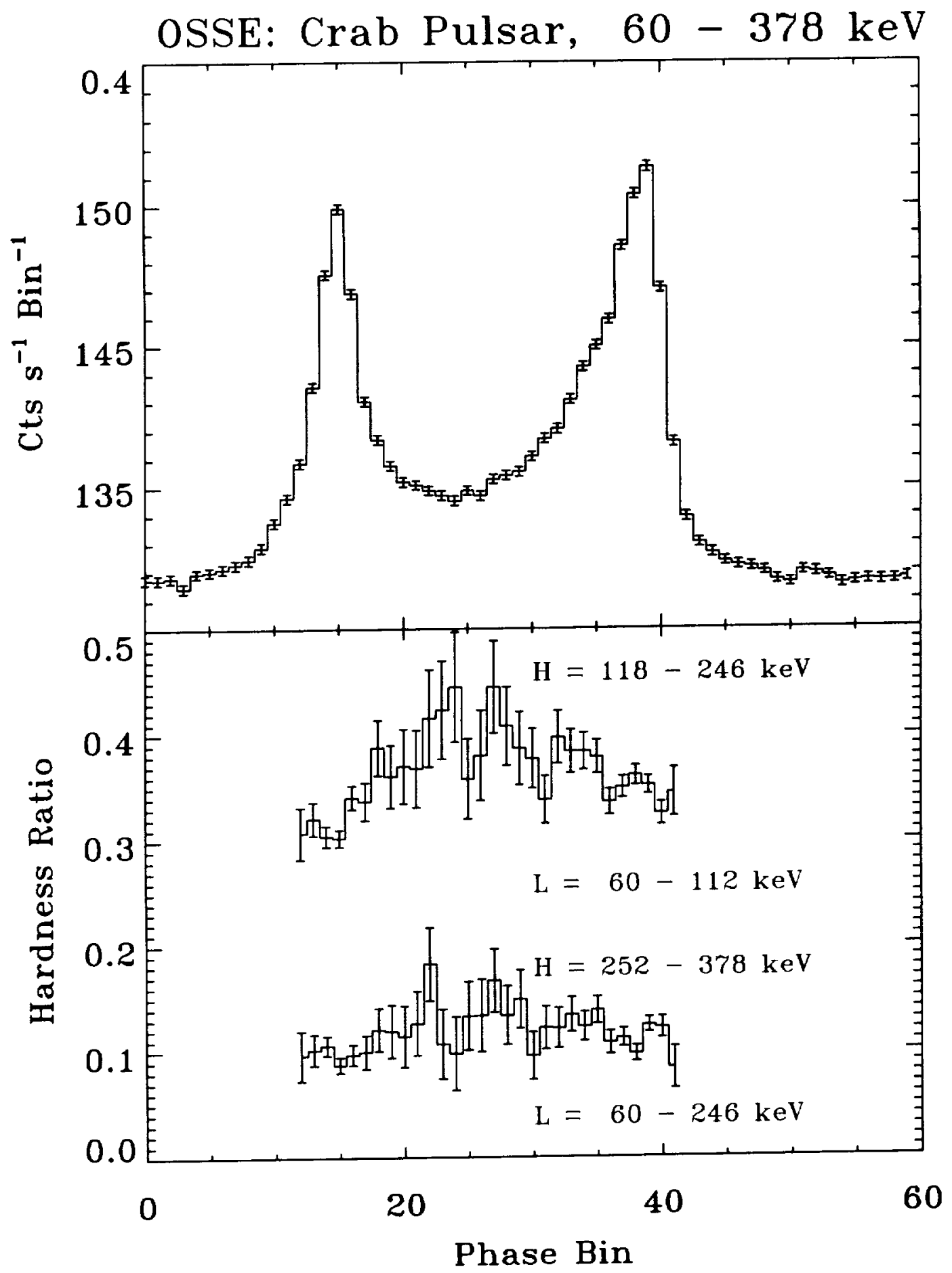

Figure 3: The Crab pulsar light curve compared with the hardness ratio, plotted as the ratio of the higher energy channel counts over the lower energy channel. 
The average spectrum is shown in Figure 4 where we see very good agreement with previous experiments. Note, this is only an approximate photon spectrum since we used a simplified instrument response model which ignored Compton scattering.

We cannot conclusively exclude the existence of the $440 \mathrm{keV}$ line in the spectrum of the second peak reported by the FIGARO group (Agrinier et al. 1990, Massaro et al. 1991) of $0.86 \pm 0.33 \times 10^{-4}$ photons $\mathrm{cm}^{-2} \mathrm{~s}^{-1}$. The FIGARO result is based on combining data from balloon flights made on 11 July 1986 and 9 July 1990. In our data, we find that no features are obvious at $440 \mathrm{keV}$ or elsewhere. Our $2 \sigma$ upper limit to a line that is intrinsically much narrower than OSSE's $\sim 8.5 \%$ detector resolution at $440 \mathrm{keV}$ is $\sim 0.8 \times 10^{-4}$ photons $\mathrm{cm}^{-2}$ $\mathrm{s}^{-1}$. On average, between 200 and $500 \mathrm{keV}$, our $2 \sigma$ sensitivity to a narrow line for this observation is $\sim 10^{-4}$ photons $\mathrm{cm}^{-2} \mathrm{~s}^{-1}$.

\section{CONCLUSIONS}

The OSSE pulsar mode is performing as designed. For the Crab pulsar, we find general agreement with previous experiments in terms of average spectra and pulse shape. We do not yet have any evidence for line features in the spectra, but further analysis must be done. The difference in spectral shape between the interpulse region and the peaks exhibited in our data is consistent with that reported by Knight (1982). This spectral difference suggests that the interpulse emission is coming from a different site than the peak emission. Comparison with concurrent radio observations made by Phase I Guest Investigators from Cornell University may help elucidate the nature of the emission sites (Lundgren and Cordes 1991).

\section{REFERENCES}

Agrinier, B., et al., 1990, Ap. J. Letters, 355, 645.

Johnson, W.N., et al., 1989, Proc. of the GRO Science Workshop, ed. W.N. Johnson, pp. 2-22.

Knight, F.K., 1982, Ap. J., 260, 578.

Lundgren, S., and Cordes, J.M., 1991, this proceeding.

Mahoney, W.A., Ling, J.C., and Jacobson, A.S., 1984, Ap. J., 278, 784.

Massaro, E., et al., 1991, Ap. J. Letters, in press.

Taylor, J.H., and Weisberg, J.M., 1989, Ap. J., 345, 434. 


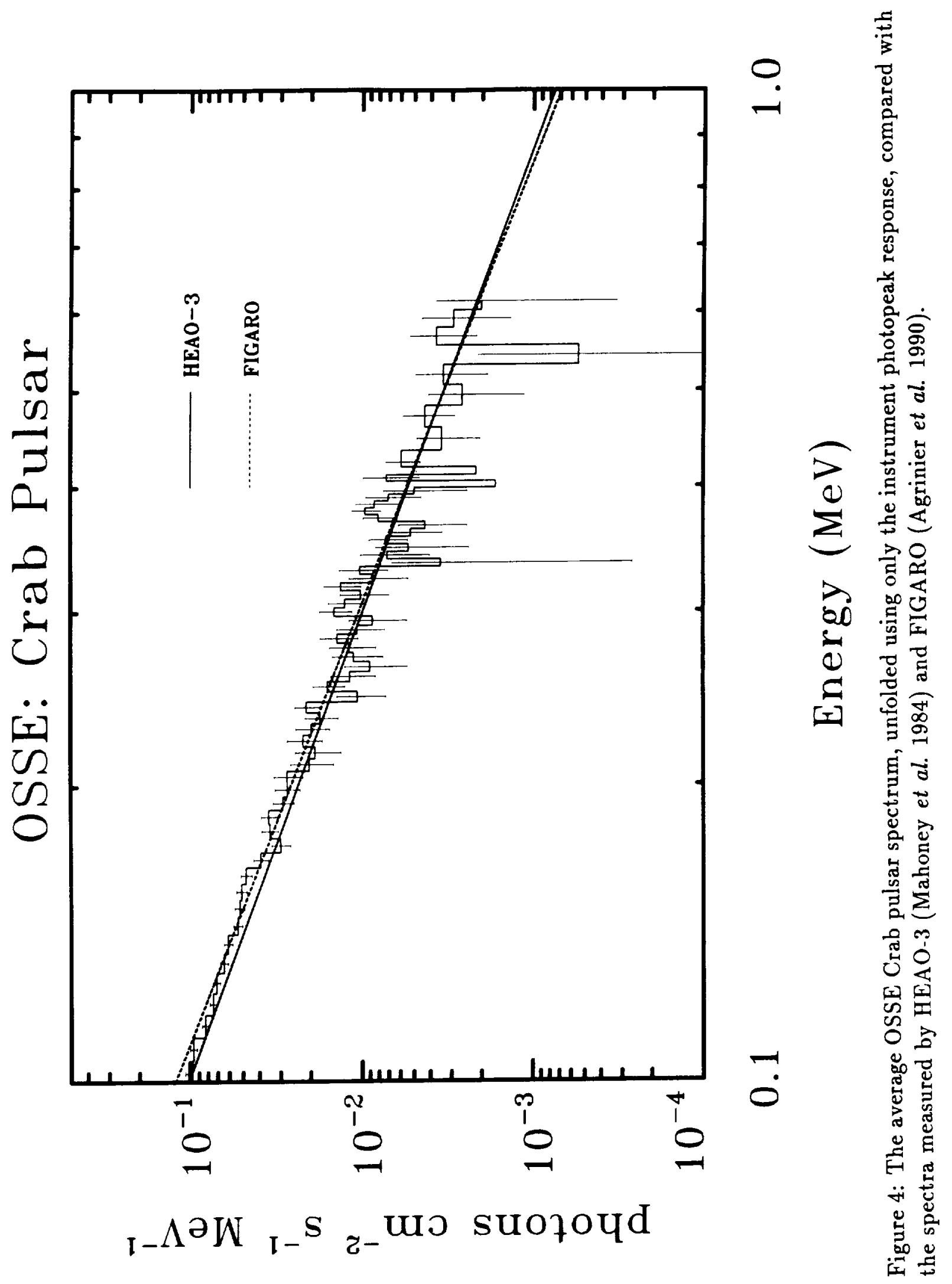

